\title{
Off-line Signature Verification based on the Modified Direction Feature
}

\author{
Stephane Armand, Michael Blumenstein and Vallipuram Muthukkumarasamy \\ School of Information and Communication Technology, \\ Griffith University, Queensland, Australia \\ Stephane.Armand@student.griffith.edu.au, \{M.Blumenstein,V.Muthu\}@griffith.edu.au
}

\begin{abstract}
Signature identification and verification has been a topic of interest and importance for many years in the area of biometrics. In this paper we present an effective method to perform off-line signature verification and identification. To commence the process, the signature's contour is first determined from its binary representation. Unique structural features are subsequently extracted from the signature's contour through the use of a novel combination of the Modified Direction Feature (MDF) in conjunction with additional distinguishing features to train and test two Neural Network-based classifiers. A Resilient Back Propagation neural network and a Radial Basis Function neural network were compared. Using a publicly available database of 2106 signatures containing 936 genuine and 1170 forgeries, we obtained a verification rate of $91.12 \%$.
\end{abstract}

\section{Introduction}

Within the field of human identification, the usage of biometrics is growing because of its unique properties such as hand geometry, iris scan, fingerprints or DNA. The use of signatures has been one of the more convenient methods for the identification and verification of human beings. A signature is also a biometric, but a behavioral one, as it can change depending on many elements such as: mood, fatigue, etc. The challenging aspects of automated signature identification and verification have been for a long time a true motivation for researchers. Research into signature verification has been vigorously pursued for a number of years [1] and is still being explored (especially in the off-line mode) [2]. On-line verification must be differentiated from off-line verification, as the number of features, which may be extracted from on-line mediums, exceed those obtained from off-line verification i.e. time, pressure and speed can be extracted from on-line modes of verification [3]. Previous approaches, such as that based on fuzzy modeling and the employment of the Takagi-Sugeno model, have been proposed using angle features extracted from a box approach to verify and identify signatures [4]. Also, The GSC (Gradient, Structural and Concavity) feature extractor provided results as high as: $78 \%$ for verification and $93 \%$ for identification [5]. Various classifiers, such as Support Vector Machines (SVMs) and Hidden Markov Models (HMMs), have also been successful in off-line signature verification; SVMs providing an overall better result than the HMM-based approach [6].

Previous work using the Modified Direction Feature (MDF) generated encouraging results, reaching an accuracy of $81.58 \%$ for cursive handwritten character recognition [7]. As an extension to previous work, the research in this paper adapts, extends and investigates MDF with signature images. The verification process implies the usage of forged signatures, discriminating the genuine from the forged. In this paper, we present experimental results for signature verification using MDF, and propose some modifications of the features extracted.

\section{Methodology}

To perform verification or identification of a signature, several steps must be performed. After preprocessing all signatures from the database by converting them to a portable bitmap (PBM) format, their boundaries are extracted to facilitate the extraction of features using MDF. Identification and verification experiments were performed with neuralbased classifiers.

\subsection{Signature Database}

Experiments have been performed with the "Grupo de Procesado Digital de Senales" (GPDS) signature database [8]. The results provided in this research used a total of 2106 signatures.

From those 2106 signatures, we used 39 sets of signatures (i.e. from 39 different persons) and, for each 
set, 24 samples of genuine and 30 samples of forgeries. Although the full GPDS database includes 140 signature sets, only a component (39 sets) of that was made available at the time of the experimentation.

\subsection{Boundary Extraction}

The boundary of each signature must be extracted prior to the feature extraction process. The binary image of each signature is preprocessed whereby the contour is extracted, providing the first step in the procedure of reducing the amount of data describing each pattern.

\subsection{Feature Extraction with MDF}

The features extracted must be appropriate for both the application and the classifier used. MDF has been used to extract features for the current problem [7]. This technique employs a hybrid of two other feature extraction techniques, Direction Feature (DF) and the Transition Feature (TF).

DF extracts direction transitions (DT), based on the replacement of the foreground pixels by their direction values. The feature vector is extracted by zoning and computing the most representative direction values in a given zone.

$\mathrm{TF}$ records the locations of the transitions (LTs) between foreground (1s) and background (0s) in binary digital images. The image is traversed from the following directions: left to right, right to left, top to bottom and bottom to top. Each time a change from ' 0 ' to ' 1 ' or from ' 1 ' to ' 0 ' occurs, the ratio between the location of the transition and the length/width of the image traversed is recorded as a feature. An averaging algorithm is used to obtain a feature vector of appropriate size in order to decrease the training/classification time.

MDF is based on the extraction technique present in TF. Not only are the locations of the transitions calculated, but also the corresponding direction values are determined, facilitating the storage of LTs and DTs. The width to height ratio feature was also included to comprise MDF. Previously, MDF was used

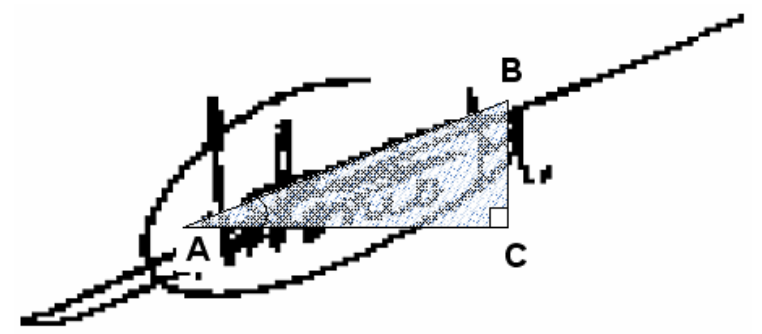

Figure 1: Extraction of the 'centroid' features with an alternate database for the problem of character recognition [7] showing encouraging performance.

\subsection{The Centroid Feature}

Another feature was considered in this research relating to the dominant angle of the signature, the 'centroid'.

First, the signature image was separated into two equal parts. The position of the centre of gravity in each part was calculated ( $\mathrm{A}$ and $\mathrm{B}$ in Figure 1). The angle between the horizontal axis and the line obtained by linking the two centres of gravity was the feature added. To convert this angle in the range of 0 to 1 , calculations were performed with the coordinates of the centres of gravity. A right triangle was considered using the centres of gravity as the extreme points of its hypotenuse, the row of the first centre of gravity and the column of the other, intersecting to form the right angle.

To obtain the 'centroid' feature, the angle $\alpha$ was first considered in the left part of the triangle, and then a calculation was performed, in order to restrict the value in the range of 0 and 1. Equations (1) and (2) are followed to get this value. The concept is shown in Figure 1. The angle $\alpha$ is illustrated at point A.

$$
\begin{aligned}
& \alpha=\frac{\arcsin \left(\frac{\text { height }}{\text { hypotenuse }}\right) \times 180}{\pi} \\
& \text { centroid }=\frac{\alpha+90}{180}
\end{aligned}
$$

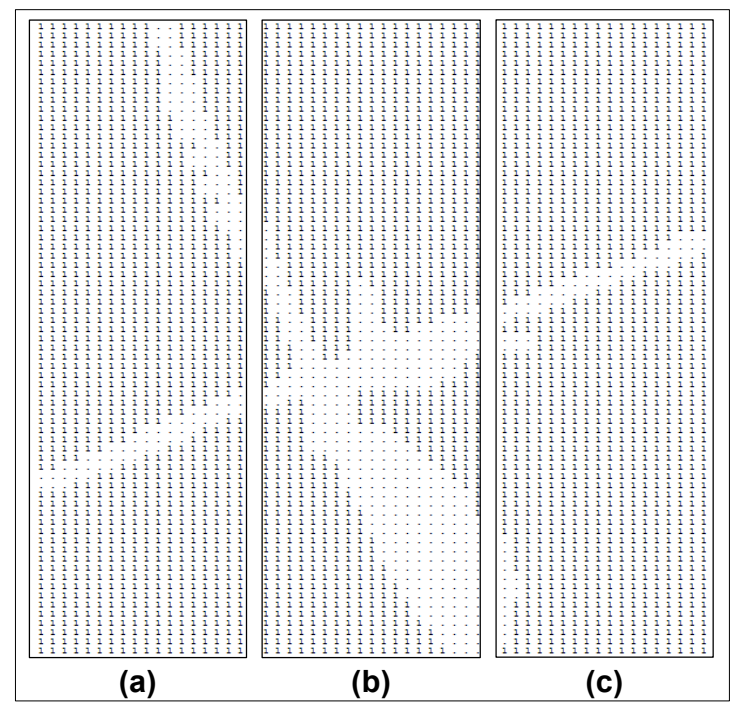

Figure 2: Extraction of the 'triSurface' feature 


\subsection{The triSurface Feature}

The surface area of two visually different signatures could be the same. For the purpose of increasing the accuracy of a feature describing the surface area of a signature, the 'triSurface' feature was implemented, as an extension, in which the signature was separated into three equal parts, vertically. Figure 2 illustrates this concept. The surface area feature is the surface covered by the signature, including the holes contained in it. The number of black pixels in the surface was counted, and the proportion of the signature's surface over the total surface of the image was calculated.

This process was used for the three equal parts of the signature, giving three values between 0 and 1 .

\subsection{The Length Feature}

The length feature represents the length of the signature, after scaling all the signatures from the database to the same height. In order to obtain a value between 0 and 1 , the minimum and the maximum signature lengths were respectively considered as 0 and 1. The remaining signature lengths were then converted to values between this minimum and maximum range.

\subsection{Neural Network Classifiers}

Two neural network classifiers were used to classify the signatures: the resilient backpropagation (RBP) neural network and the Radial Basis Function (RBF) network. The database was split in two parts, to perform the training and testing components. From the genuine set, 18 samples of each signature were used for training, and 6 for testing. We used 22 samples of each signature for training the forged signatures and 8 for testing purposes. Parameters such as the number of iterations and hidden units were varied extensively for

Table 1. Results using the RBP NN classifier

\begin{tabular}{c|cccc|c} 
& \multicolumn{5}{c}{ Verification Rate [\%] } \\
\cline { 2 - 6 } & Set 1 & Set 2 & Set 3 & Set 4 & Sets Avg. \\
\hline MDF & 83.33 & 88.64 & 87.18 & 85.16 & 86.08 \\
MDF-C & 84.07 & 87.91 & 89.56 & 87.73 & 87.32 \\
MDF-T & 85.53 & 86.45 & 87.55 & 87.91 & 86.86 \\
MDF-L & 84.43 & 88.10 & 89.01 & 85.35 & 86.72 \\
MDF-CT & 84.43 & 87.36 & 88.46 & 89.56 & 87.45 \\
MDF-CL & 82.23 & 87.73 & 84.98 & 86.45 & 85.35 \\
MDF-TL & 82.97 & 86.63 & 87.18 & 89.19 & 86.49 \\
MDF-CTL & 81.68 & 87.36 & 87.36 & 87.18 & 85.90 \\
\hline
\end{tabular}

RBP during experimentation, also the number centres was varied between 40 and 10000 for the RBF network.

\section{Experiments}

The signatures were firstly converted into a Portable Bitmap (PBM) format in order to facilitate processing as binary images. Subsequently, their boundaries were extracted for further processing. An adapted version of MDF was used to extract the features described earlier. The features extracted were values scaled between 0 and 1, to facilitate $\mathrm{NN}$ training for experimentation. C, Java and the Matlab environment were used for implementing/investigating the proposed techniques.

\subsection{Verification with MDF}

Using MDF, a total of 121 features were obtained. Modifications of the MDF technique have been proposed to improve classification accuracy, with the hypothesis that adding distinguishing features could improve the verification rate. Experiments with MDF merged with each new feature, or a combination of new features were conducted separately, increasing the feature vector up to 126 inputs. Some signatures are challenging to verify, as the variation of a signature from someone could be highly contrasted.

\subsection{4-fold Validation}

Four-fold cross validation was used in this research. The signatures from the database were partitioned into four equal sections in their related type (genuine or forged). Thus, four sections for the genuine, and four sections for the forged, resulted from this partition. Three sections of each were used for training, and testing was performed with the remaining unseen

Table 2. Results using the RBF NN classifier

\begin{tabular}{c|cccc|c}
\cline { 2 - 6 } & \multicolumn{5}{c}{ Verification Rate [\%] } \\
\hline MDF & 87.91 & 90.48 & 89.74 & 90.29 & 89.61 \\
MDF-C & 88.10 & 89.74 & 90.11 & 89.93 & 89.47 \\
MDF-T & 87.73 & 90.48 & 89.93 & 90.11 & 89.56 \\
MDF-L & 89.01 & 91.21 & 90.66 & 90.66 & 90.38 \\
MDF-CT & 88.28 & 90.29 & 90.11 & 89.74 & 89.61 \\
MDF-CL & 88.10 & 90.84 & 89.74 & 90.29 & 89.74 \\
MDF-TL & 89.38 & 87.91 & 89.38 & 88.46 & 88.78 \\
MDF-CTL & 89.19 & 91.76 & 91.94 & 91.58 & 91.12 \\
\hline
\end{tabular}


Table 3. Comparison between RBP and RBF neural network classifiers

\begin{tabular}{l|cccccccc}
\cline { 2 - 8 } & \multicolumn{10}{c}{ MDF } & MDF-C & MDF-T & MDF-L & MDF-CT & MDF-CL & MDF-TL & MDF-CTL \\
\hline RBP & 86.08 & 87.32 & 86.86 & 86.72 & 87.45 & 85.35 & 86.49 & 85.90 \\
RBF & 89.61 & 89.47 & 89.56 & 90.38 & 89.61 & 89.74 & 88.78 & 91.12 \\
\hline
\end{tabular}

signature sets. As a result, four training and test sets were obtained, and experiments were run four times with the different sets, obtaining varying results. By doing so, the results for signature verification were suitably validated.

\section{Results and Discussion}

The verification process is different from identification, as forged signatures are also part of the database in the former; the classifier was required to distinguish between genuine and forged signatures. To conduct verification, 1560 signatures were used for training and 546 signatures for testing. However, in this research, verification includes identification. Instead of only accepting or rejecting a signature, the classifier can also output the signature class in which the tested signature belongs to.

This section describes the results obtained experimenting as follows: MDF in its original version, or with one or more of the extra features: centroid (C), triSurface (T), length (L).

As shown in Table 1 and Table 2, MDF merged with other features outperformed the original version of MDF, for most of the experiments.

\subsection{Comparison between RBP and RBF}

Table 3 displays the comparison between RBP and RBF. Using the RBF classifier, the results obtained were better overall in comparison with the RBP classifier. Having experimented only with part of the GPDS database, a direct comparison with the research of Martinez et al. [8] is not feasible. However, in a qualitative sense, this work compared well with their research.

\section{Conclusions}

The principal objective of this paper was to investigate the efficiency of the feature extractor MDF for signature verification. Investigations adding new feature values to MDF were performed, assessing the impact on the verification rate of the signatures, using 4-fold cross validation. Two different neural classifiers were used. Most of the experiments conducted with MDF merged with the new features obtained better results than the original MDF. Using RBP, MDF reached an $86.08 \%$ v. r., and MDF-CT reached $87.45 \%$. The RBF classifier provided better results than the RBP classifier. The best v. r. obtained reached $91.12 \%$ with MDF-CTL, the combination of all the features described in this paper.

In future research, investigations will be conducted to enhance the feature extraction process, including further combinations and investigations of the features. In addition, a larger signature database will be collected, including multilingual signatures, to investigate the techniques proposed in this paper.

\section{References}

[1] K. Han, and I.K. Sethi, "Handwritten Signature Retrieval and Identification", Pattern Recognition 17, 1996, pp. 83-90.

[2] S. Chen, and S. Srihari, "Use of Exterior Contour and Shape Features in Off-line Signature Verification", 8th International Conference on Document Analysis and Recognition (ICDAR '05), 2005, pp. 1280-1284.

[3] A. Kholmatov, and B. Yanikoglu, "Identity Authentication using improved online signature verification method", Pattern Recognition Letters, 2005, in press.

[4] M. Hanmandlu, M.H.M. Yusof, and V.K. Madasu, "Offline Signature Verification using Fuzzy Modeling", Pattern Recognition 38, 2005, pp. 341-356.

[5] M.K. Kalera, S. Srihari, and A. Xu, "Off-line signature verification and identification using distance statistics", International Journal of Pattern Recognition and Artificial Intelligence 18(7), 2004, pp. 1339-1360.

[6] E.J.R. Justino, F. Bortolozzi, and R. Sabourin. "A comparison of SVM and HMM classifiers in the off-line signature verification", Pattern Recognition Letters 26, 2005, pp. 1377-1385.

[7] M. Blumenstein, X.Y. Liu, and B. Verma, "A Modified Direction Feature for Cursive Character Recognition", International Joint Conference on Neural Networks (IJCNN '04), 2004, pp. 2983-2987.

[8] L.E. Martinez, C.M. Travieso, J.B. Alonso, and M. Ferrer, "Parametrization of a forgery Handwritten Signature Verification using SVM", IEEE 38th Annual 2004 International Carnahan Conference on Security Technology, 2004, pp. 193-196. 